\section{Correspondence on "European League Against Rheumatism (EULAR)/American College of Rheumatology (ACR) SLE classification criteria item performance" by Aringer et al}

Antinuclear antibodies (ANA) are important laboratory markers for the diagnosis and classification of systemic lupus erythematosus (SLE). In the 2019 European League Against Rheumatism (EULAR)/American College of Rheumatology (ACR) classification criteria for SLE, ANA with tit $\geq 1: 80$ are an entry criterion. ${ }^{1}$

Even though ANA 1:80 are highly sensitive for SLE, they have a low specificity. This has recently been reinforced by Aringer et $a l^{2}$ who analysed the performance of the individual items included in the 2019 EULAR/ACR classification criteria for SLE on a large group of patients with SLE $(n=1197)$ and nonSLE disease controls $(n=1074)$, including patients with other connective tissue diseases (two-thirds of the controls). In this study, ANA with tit $\geq 1: 80$ were highly sensitive (99.5\%), but only $19.4 \%$ specific for SLE. ${ }^{2}$ As ANA are an entry criterion for the 2019 EULAR/ACR classification criteria, the low specificity of ANA for SLE does not affect the specificity of the 2019 SLE classification criteria. An important item that conferred specificity to the 2019 SLE classification was the attribution rule. ${ }^{2}$ The authors stressed that ANA are a useful screening test, but not specific and that the classification criteria should not be used as diagnostic criteria. ${ }^{2}$

As ANA are employed in the context of both classification and diagnosis, it is important to get better insights into the interpretation of ANA test results. We here argue that interpretation of ANA test results can be improved by assigning titre-specific likelihood ratios (LR)s. The LR is a good indicator of the clinical value of a test result. It is the ratio of the fraction of patients with a particular test results to the fraction of controls with the same test result. For example, a test result with an LR of 5 indicates that such result is 5 times more likely in patients than in controls.

Positioning of ANA as an entry criterion in the 2019 EULAR/ ACR classification was based on a literature review and metaregression analysis on the performance of indirect immunofluorescence (IIF) on HEp-2 substrates. ${ }^{3}$ In the paper that describes this metaregression analysis (including 13080 patients with SLE and 7539 controls), data on sensitivity, specificity and negative and positive LR of ANA for SLE are given for different cutoffs $\left(1: 40,1: 80,1: 160\right.$ and 1:320). ${ }^{3}$ Figure 1 (left hand part) graphically recapitulates the results of the metaregression analysis. ANA at a cut-off titre of 1:80 had a high sensitivity $(97.8 \%) .{ }^{3}$ The specificity and positive LR were 74.7 and 3.87, respectively. ${ }^{3}$ Because of the high sensitivity, ANA at 1:80 cut-off by IIF was positioned as an entry criterion for the SLE classification. $^{3}$

The LRs reported by Leuchten et $a l^{3}$ are for single cut-off values, implying that all values higher than the cut-off value are considered equal. For example, for a 1:80 cut-off, all patients with ANA $\geq 1: 80$ are grouped (ie, 1:80, 1:160, 1:320, 1:640, ...). Such approach does not take into account the fact that the likelihood for disease increases with increasing antibody levels. Conversely, titre-specific information gives more detailed information on the clinical value of a specific result. Based on the data provided by Leuchten $e t a l,{ }^{3}$ we calculated the titre-specific LRs for a negative, 1:40, 1:80 and 1:160 test result (figure 1, righthand panel). The titre-specific LRs were 0.02, 0.08, 0.17 and 0.94 , respectively. These titre-specific LRs are clearly lower than the LRs calculated using a dichotomous approach as reported by Leuchten et $a l^{3}$ the reason being that a dichotomous approach groups all values exceeding the cut-off. For example, the titrespecific LR for 1:80 was 0.17 , whereas the positive LR associated with a single 1:80 cut-off was 3.87 . Of note, the LR associated with a single 1:80 cut-off reported in the recent study by Aringer et $a l^{3}$ was 1.23 . 
A test result with an LR of 0.17 (the 1:80 titre-specific LR) is 5.8 times more likely to be found in non-SLE than in SLE. Thus, even though an ANA with titre 1:80 is of use as entry criterion for classification, it is not supportive of a diagnosis of SLE (it rather makes this diagnosis less likely). According to the meta-analysis of Leuchten et al, ${ }^{3} 2 \%$ of patients with SLE (and $11.5 \%$ of controls) had a 1:80 ANA test result. The titre-specific LR increases with increasing titres and was 0.94 for a titre $1: 160$ and 25.29 for titres $1: \geq 320$. Thus, the strength of ANA in supporting an SLE diagnosis increases with the increase in the antibody titre. Providing clinicians with such information will help them with the interpretation of ANA test results. However, there might be manufacturer-dependent differences between HEp-2 substrates. ${ }^{4}$ Consequently, titre-specific LR may have to be established for each HEp-2 substrate.

In addition to the titre, the IIF antibody pattern will likely provide additional information. Some patterns are associated with certain specific antibodies (and diseases) (eg, a homogeneous pattern is found in patients with SLE with antibodies to dsDNA, -histones or nucleosomes). ${ }^{5-7}$ The only unambiguous association is for anticentromere antibodies with anticentromere positive (limited cutaneous) systemic sclerosis.

To conclude, we support the statement of Aringer et al that the classification criteria for SLE should not be used for diagnosis. A dichotomous interpretation of ANA test results overlooks the fact that the likelihood for SLE increases with increasing antibody level. Providing titre-specific LRs for ANA may be one way to help clinicians with the interpretation of ANA.

\section{Xavier Bossuyt $\odot{ }^{1}{ }^{1}$ Walter Fierz, ${ }^{2}$ Pier Luigi Meroni ${ }^{3}$ \\ ${ }^{1}$ Department of Microbiology, Immunology and Transplantation, KU Leuven, and Department of Laboratory Medicine, University Hospitals Leuven, Leuven, Belgium ${ }^{2}$ Schweizerischer Verband der Diagnostikindustrie, Bern, Switzerland ${ }^{3}$ Experimental Laboratory of Immunological and Rheumatologic Researches, Istituto Auxologico Italiano Istituto di Ricovero e Cura a Carattere Scientifico, Milano, Italy}

Correspondence to Dr Xavier Bossuyt, KU Leuven University Hospitals Leuven, Leuven, Flanders, Belgium; xavier.bossuyt@uzleuven.be

\section{Handling editor Josef S Smolen}

Acknowledgements We thank Martin Aringer for critically reading the manuscript.

Contributors XB drafted the manuscript. All authors discussed the content and contributed to the final manuscript.
Funding The authors have not declared a specific grant for this research from any funding agency in the public, commercial or not-for-profit sectors.

Competing interests None declared.

Patient and public involvement Patients and/or the public were not involved in the design, or conduct, or reporting, or dissemination plans of this research.

Patient consent for publication Not required.

Provenance and peer review Commissioned; internally peer reviewed.

(C) Author(s) (or their employer(s)) 2021. No commercial re-use. See rights and permissions. Published by BMJ.

\section{Check for updates}

To cite Bossuyt X, Fierz W, Meroni PL. Ann Rheum Dis Epub ahead of print: [please include Day Month Year]. doi:10.1136/annrheumdis-2021-221288

Received 16 August 2021

Accepted 17 August 2021

\section{Linked}

http://dx.doi.org/10.1136/annrheumdis-2021-221374

Ann Rheum Dis 2021;0:1-2. doi:10.1136/annrheumdis-2021-221288

\section{ORCID iDs}

Xavier Bossuyt http://orcid.org/0000-0001-6856-8485

Pier Luigi Meroni http://orcid.org/0000-0002-3394-1451

\section{REFERENCES}

1 Aringer M, Costenbader K, Daikh D. European League against Rheumatism/American College of rheumatology classification criteria for systemic lupus erythematosus. Ann Rheum Dis 2019;2019:1151-9.

2 Aringer M, Brinks R, Dörner T, et al. European League Against Rheumatism (EULAR)/ American College of Rheumatology (ACR) SLE classification criteria item performance. Ann Rheum Dis 2021:annrheumdis-2020-219373.

3 Leuchten N, Hoyer A, Brinks R. Systemic lupus erythematosus classification criteria Steering Committee. performance of antinuclear antibodies for classifying systemic lupus erythematosus: a systematic literature review and meta-regression of diagnostic data. Arthritis Care Res 2018;70:428-38.

4 Pisetsky DS, Spencer DM, Lipsky PE, et al. Assay variation in the detection of antinuclear antibodies in the sera of patients with established SLE. Ann Rheum Dis 2018;77:911-913

5 Willems P, De Langhe E, Westhovens $R$, et al. Antinuclear antibody as entry criterion for classification of systemic lupus erythematosus: pitfalls and opportunities. Ann Rheum Dis 2019;78:e76.

6 Vulsteke J-B, Van Hoovels L, Willems P, et al. Titre-specific positive predictive value of antinuclear antibody patterns. Ann Rheum Dis 2019:annrheumdis-2019-216245.

7 Bossuyt $\mathrm{X}$, De Langhe E, Borghi MO, et al. Understanding and interpreting antinuclear antibody tests in systemic rheumatic diseases. Nat Rev Rheumatol 2020;16:715-26. 